\title{
Mathematical Modeling of Failure and Deformation Processes in Metal Alloys and Composites
}

\author{
Mark Petrov \\ Department of Strength and Longevity of Materials and Structural Components, Siberian Aeronautical Research Institute Named After S. A. \\ Chaplygin, Novosibirsk, Russia \\ Email address: \\ mark-st@ngs.ru

\section{To cite this article:} \\ Mark Petrov. Mathematical Modeling of Failure and Deformation Processes in Metal Alloys and Composites. American Journal of Physics and \\ Applications. Vol. 8, No. 4, 2020, pp. 46-55. doi: 10.11648/j.ajpa.20200804.11
}

Received: May 13, 2020; Accepted: July 3, 2020; Published: July 17, 2020

\begin{abstract}
Based on experimental examples, the strength characteristics of metal alloys and composites under tensile and compressive loads are considered to demonstrate both their similarity and difference. Under tensile loads, their behavior is essentially the same. Under compressive loads, the composite shows different properties, but similar to the behavior of a metal alloy under tension. When tensioned and compressed, it fractured as a material with a different structure. When a metal alloy is cyclically compressed, the damage accumulation process is attenuated, which reduces the alloy longevity during subsequent tension. The analysis of experimental data for various types of loading from the standpoint of the kinetic concept of fracture is carried out. Instead of a number of incompatible approaches or a formal description of experimental data, that based on the theory of reaction rates is used. Mathematical modeling of processes is carried out using rheological models of the material. Structural models of the material, called physical media, reflect the thermodynamic processes of flow, failure, and changes in the structure of the material. Parametric identification of structural models is carried out on the basis of the minimum necessary basic experiment: loading of specimens with different speeds at several temperature values and by the amplitude dependence of inelasticity. Based on results of these experiments, the scope of applicability conditions for this material and test modes necessary for parametric identification of models are selected. One fracture criterion is used, which formally corresponds to the achievement of a threshold concentration of micro-damage in any volume of the material, leading to macro-fracture. The application of mathematical models for calculating the longevity of materials depending on the temperature and force loading conditions and the nature of their changes is shown. Calculations of longevity under constant, monotonously increasing and variable loads under conditions of constant or changing temperatures are based on the relationship of plastic flow and failure processes distributed over the volume of the material. They are performed numerically by time steps depending on the ratio of the rate of change of temperature and stresses.
\end{abstract}

Keywords: Creep, Fatigue, Damage, Thermal Activation Analysis, Inelasticity, Rheology, Mathematical Modeling

\section{Introduction}

There are three methods to assess the longevity of structures [1]. The first (most rigorous) one is a detailed study of the kinetics of deformation and fracture in a wide range of external conditions and the development of a model reflecting the main physical regularities in the behavior of the loaded material. The second (simpler) method of engineering calculations is based on the methods of limiting elastic-plastic analysis and employs simpler models. It can also include methods of deformable solid mechanics that use experimentally determined relationships between stresses, strains and longevity. The third method is the simplest, but also the least accurate. It is used if there are no available data on the material properties and represents an empirical relationship of longevity with external loading conditions. Here we will talk about the first method, based on a mathematical description of general and local plastic strains that occur over time and accompany failure, which is an alternative approach that eliminates the conditionality and incompatibility of existing methods [2].

First, the structural heterogeneity of the material is modeled, which is quantitatively represented by internal stresses field arising when the material is loaded. Second, the 
thermodynamic processes of the material flow associated with the process of damage formation and development in its volumes are also simulated. The single fracture criterion is reaching a threshold damage concentration in any volume of the material, which translates the failure process to the next dimensional level (the fracture concentration criterion).

The first step in the development of a mathematical model of a material is to test material specimens at different temperatures and constant stresses or under monotonous loading at different rates. The result of processing is obtaining a force dependence of the activation energy of fracture (AEF). The model is based on the relationship between the rate of failure, the rate of deformation, and longevity.

Next, cyclic tests are carried out, and a relationship is established between inelastic deformations and endurance (or the rate of inelastic deformations and longevity), which is similar to the relationship between the flow rate and fracture of a material during creep [3]. A similar simulation based on mechanical models is known [4]. However, the models we use are fundamentally different in that they are based on the equations of physical kinetics that explicitly involve the temperature [5].

All cases of the material behavior will be considered from the standpoint of the kinetic concept of fracture $[2,3,5,6]$. Let us demonstrate experimentally observed patterns of the fracture and deformation of metal alloys and composite materials under various loads. At each type of loading, the material displays only some part of its properties. It is necessary to follow well-approved test methods and their sequence. At the same time, it is desirable to have a minimum of necessary information and the complexity of tests and see ways to further research to solve special, more complex problems.

\section{Failure Under Constant and Monotonously Increasing Loads}

As in the study of any new material, we begin with the simplest experiments in which the basic laws of the processes are visible. Figures 1 and 2 illustrate the results of the thermal activation analysis of the fracture of an aluminum alloy and carbon fiber specimens under constant and increasing loads and various temperatures. The values of $U(\sigma)$ are obtained by multiplying the universal gas constant $\mathrm{R}$ by the absolute temperature $T$ and by $\ln \left(\tau \nu_{0}\right)$, where $v_{0}$ is the characteristic Debye frequency, which is the inverse of the average period of thermal vibrations of atoms in a solid [6]. The failure time $\tau$ is determined directly from the experiment if the stress and temperature are constant, and as a value reduced to the maximum stress, calculated as an integral of the failure rate over time [2]. The linearity of the force dependence of the AEF $U_{0}-\gamma \sigma$ indicates the area of the temperature and time conditions where the material structure does not undergo significant changes.

For an aluminum alloy tested in two structural states under tension, the same value of the initial AEF $U_{0}$ is obtained, and the difference is in the different slopes of the force dependence of the AEF, where the constancy of the activation volume $\gamma$ will means the stability of the material structure under these loading conditions. The alloy at a later stage of decay of a supersaturated metal solid solution shows lower strength characteristics (increase $\gamma$ ).

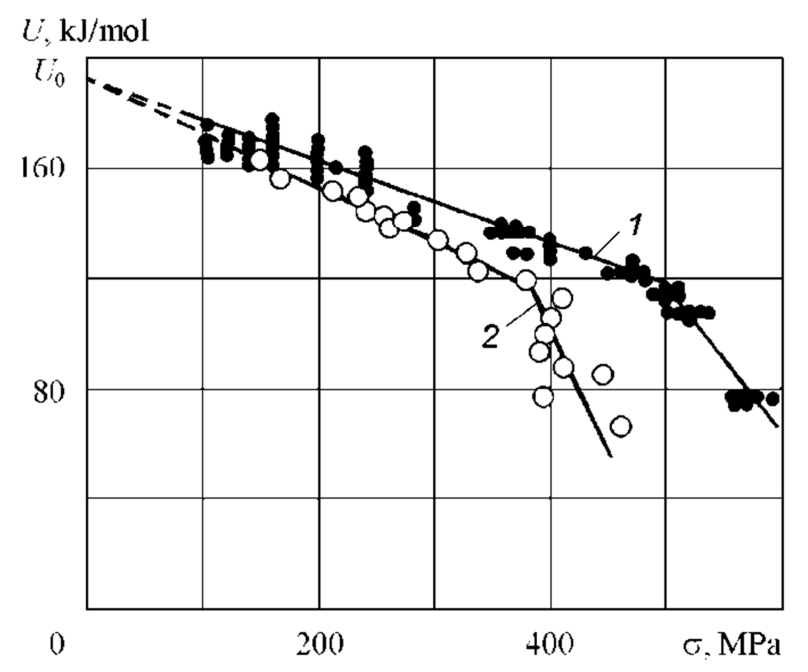

Figure 1. Force dependences of AEF of in specimens of AK4-1 T1 alloy in the aged at maximum hardness (1) and over-aged (2) states under tension [2]: 1 constant loads in the temperature range of $130-175^{\circ} \mathrm{C}$ (longevity of 3030,000 hours), 2 - monotonously increasing loads in the temperature range of $20-340{ }^{\circ} \mathrm{C}$ (loading time $\approx 30 \mathrm{~s}$ ).

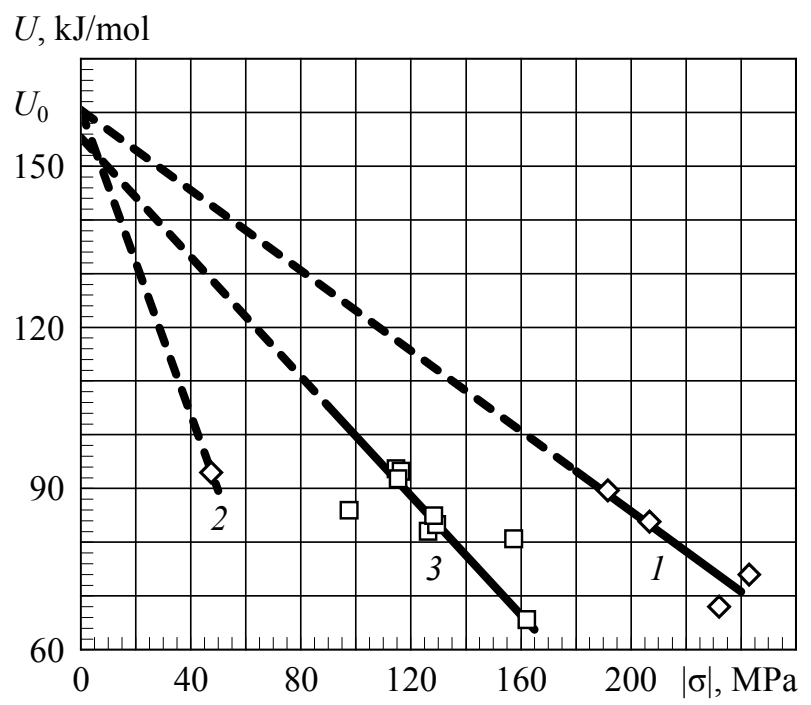

Figure 2. Force dependences of AEF of T700 carbon fiber specimens of two types under monotonously increasing compressive loads; temperature, ${ }^{\circ} \mathrm{C}$ : the first type 15-90 (1) and 130 (2), the second type 15-120 (3); loading time $1.5-130 \mathrm{~s}$.

The strength characteristics of carbon fiber obtained under compressive loads, when they are determined by the properties of the binder in the composite, reveals a similar dependence $U_{0}-\varkappa \sigma$ in a certain range of temperature and time conditions. The scatter of experimental data is much greater here, and the initial AEF $U_{0}$ is determined with a greater error. The types of the tested specimens differ in their reinforcement schemes, but their load-bearing capacity mainly 
depended on the number of longitudinally oriented layers of the composite, similar to the case of tension [7, 8]. Experiments at $130^{\circ} \mathrm{C}$ showed that the structure-sensitive coefficient $\gamma$ drastically increases, testifying to the transition of the binder material to another structural state (the value $U(|\sigma|)$ is connected by a straight line with $U_{0}$ ).

How will the material be fractured if the stress signs are changed to the opposite? Studies of the failure of plastic metal alloys at constant stresses show that a specific value of longevity is obtained for each combination of temperature and stress (Figure 1). If the material specimens are loaded with the same compressive stresses, the failure process continues further without any external signs of fracture [9].

After testing carbon fiber specimens with monotonously increasing tensile and compression loads, we obtained a three-fold decrease in the so-called "strength" of the material during compression, i.e., the opposite effect. This is understandable, since the composite is a structure, and the fibers cannot endure a compressive load without a binder.

\section{Failure Under Cyclic Loads}

A similar pattern is observed with cyclic loading. Tests of a metal alloy with a constant loading amplitude $\sigma_{a}$ when the load asymmetry index $a=\sigma_{m} / \sigma_{a}$ changing from +1.5 to -1.5 have shown that endurance tends to a very large value after passing to cyclic compression (Figure 3). Certainly, damage (microcracks) does appear and grow in this case, and some specimens are fractured [2]. The residual endurance of non-fractured specimens in subsequent tests with a symmetrical loading cycle was four times smaller on the average as compared to the initial state.

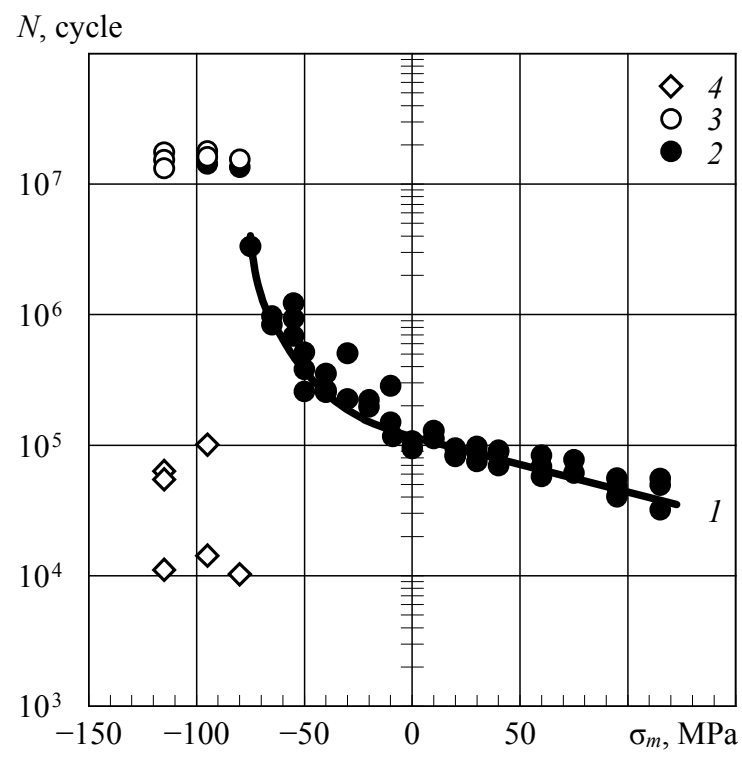

Figure 3. Endurance of 1201 AT1 alloy specimens shaped as strips with a cross section $6 \times 30 \mathrm{~mm}$ with a central hole $\varnothing 20 \mathrm{~mm}\left(K_{t}=2.08\right)$ versus the mean stress of the cycle $\sigma_{m}$ during the transition from tension to compression $\left(20{ }^{\circ} \mathrm{C}, 40 \mathrm{~Hz}\right.$, nominal stress amplitude $\left.80 \mathrm{MPa}\right): 1$ - calculation by the material model; 2 - specimens destroyed; 3 -specimens not destroyed $(N \approx$ $\left.1.4 \times 10^{7}\right) ; 4$ - residual endurance of non-destroyed specimens in the case of a symmetric loading cycle.
Curve 1 in the figure is calculated based on the fact that the local plastic strains at each time step are associated with damage accumulation only if they have a positive sign and external stresses are also tensile. In other words, we ignore the damaging effect of compression. After obtaining a calculated estimate of the longevity of the specimens based on the effective factor of strain concentration, recalculation was performed to the number of cycles that occurred during specimen fracture.

In all cases, mathematical modeling allows one to calculate an arbitrary temperature-force loading process in time without using any "equivalent" programs that replace and distort the real process and use various simplifications in each case. Figure 4 shows a structural rheological model of the material, which is used to reproduce the general and local plastic flow processes accompanying the damage development in calculations. The parameters $A$ and $B$ contain the activation parameters of flow processes: initial activation energy, activation volume, temperature, contribution of plastic strain of the element to the total strain of the material and its relationship to failure for each volume that this element represents [2].

The difference from the similar mechanical structural model of the material based on the Saint-Venant body [4] is the replacement of the dry friction element with an element that describes plastic flow kinetics $[2,5]$ :

$$
\dot{\varepsilon}=A \exp (B \sigma)
$$

which was named Zhurkov's body (symbol Zh), and

$$
\dot{\varepsilon}=2 A \sinh (B \sigma)
$$

named Kauzmann's body (symbol Km), where $A=\varepsilon_{*} v_{0} \exp \left[-Q_{0} /(R T)\right], \quad B=\alpha /(R T)$. The activation parameters of the plastic flow and failure processes for metal alloys usually coincide: $Q_{0} \approx U_{0}$ and $\alpha \approx \gamma[2-6,10,11]$. An additional structural-sensitive factor here is the parameter $\varepsilon_{*}$ which takes into account that the deformation processes are the sum of a number of local events [12].

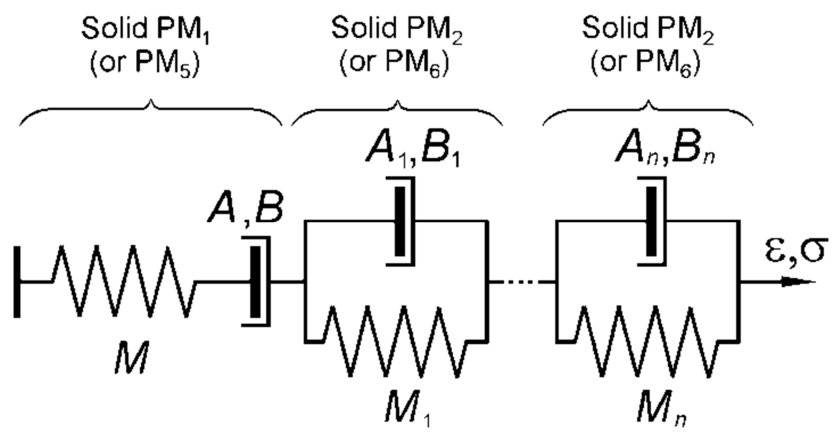

Figure 4. Structural model of a material describing the elasticity, creep, and hysteresis-type in elasticity by a set of elements with a parallel connection of an elastic body (Hooke's body) and a plastic flow body (Zhurkov's or Kauzmann's body) [2].

Each structural element of the model with a parallel connection of Hooke and, for example, Zhurkov's bodies 
(body $\mathrm{PM}_{2}$ ) has its own parameters $A, B, M$ and its own contribution to the total strain of the material [2]. At a constant loading rate $\mathrm{d} \sigma / \mathrm{d} t=D$ at the time step $t$, the solution of the differential equation of deformation of this structural element is the expression,

$$
\varepsilon=\frac{1}{M}\left[\sigma_{0}+D t+\frac{1}{B} \ln \left\{\begin{array}{l}
\exp \left[-B\left(\sigma_{0}-M \varepsilon_{0}+D t\right)\right] \\
+\frac{A M}{D}[1-\exp (-B D t)]
\end{array}\right\}\right]
$$

At $t=0$ stress $\sigma=\sigma_{0}$ and strain $\varepsilon^{=} \varepsilon_{0}$. At $D=0$ it takes the form:

$$
\varepsilon=\frac{1}{M}\left[\sigma_{0}+\frac{1}{B} \ln \left\{\exp \left[-B\left(\sigma_{0}-M \varepsilon_{0}\right)\right]+A B M t\right\}\right] .
$$

Solutions (3) and (4) are sufficient to describe any arbitrary loading process, representing it as a piecewise linear dependence.

The corresponding solutions for a sequential connection of Hooke's and Zhurkov's bodies (body $\mathrm{PM}_{1}$ ) are

$$
\varepsilon=\varepsilon_{0}+\frac{D t}{M}+A \exp \left(B \sigma_{0}\right) \frac{\exp (B D t)-1}{B D} .
$$

At $D=0$, this dependence transforms to the creep equation

$$
\varepsilon=\varepsilon_{0}+A \exp \left(B \sigma_{0}\right) t
$$

In this case, the remaining elements of the model describe the component of unsteady creep as the sum of solutions (4).

By deforming the $\mathrm{PM}_{1}$ body at a constant speed ( $\mathrm{d} \varepsilon / \mathrm{d} t=C$ ), we obtain the stress-strain relationship

$$
\sigma=-\frac{1}{B} \ln \left\{\exp \left[-B\left(\sigma_{0}+M C t\right)\right]+\frac{A}{C}[1-\exp (-B M C t)]\right\} .
$$

As the time progresses $(t \rightarrow \infty)$, this expression yields the flow stress

$$
\sigma=-\frac{1}{B} \ln \left(\frac{A}{C}\right)
$$

which depends on the strain rate and temperature (the so-called "yield strength"). If the loading terminated at some time instant $\left(t=0, \sigma=\sigma_{0}, \mathrm{~d} \varepsilon / \mathrm{d} t=0\right)$, we obtain the stress relaxation equation

$$
\sigma=-\frac{1}{B} \ln \left[\exp \left(-B \sigma_{0}\right)+A B M t\right] .
$$

Solution (4) similarly describes the relaxation of internal stresses in a structural element. If the entire sequence of model elements shown in Figure 4 is deformed at a constant rate, we can apply the method of successive approximations and obtain a result known as "plasticity with hardening." Constructing the stress-strain dependence from solutions (5) and (7), we see that two "plasticity theories" are obtained from the solution of the same differential creep equation.

The structural elements of the material model indicated by the symbols $\mathrm{PM}_{5}$ and $\mathrm{PM}_{6}$ (PM stands for Physical Medium), when using the Kauzmann body (2) lead to more complex solutions, which however, yield the same numerical result at high stresses [5]. It is advisable to use them at low stresses and high temperatures for a more accurate description of flow and failure rates.

The quantitative characteristic of the material structure is represented by the internal stress field that occurs under load. When modeling the deformation process distributed over local volumes of the material, a discrete representation of the field is used in accordance with the structural model (Figure 4) and the amplitude dependence of inelasticity [7, 10]. Loading carbon fiber with a symmetrical cycle, we see that, in contrast to a metal alloy, the increase in inelastic strain occurs mainly in the compression half-cycle [10], and the largest increase is observed in the first half-cycle. Figure 5 shows the amplitude dependence of the disclosure of the hysteresis loop in the case of loading of one of the previously tested specimens [10]. The relaxation part of inelastic strain, which is also present in composites, is here subtracted from the total inelastic strain.

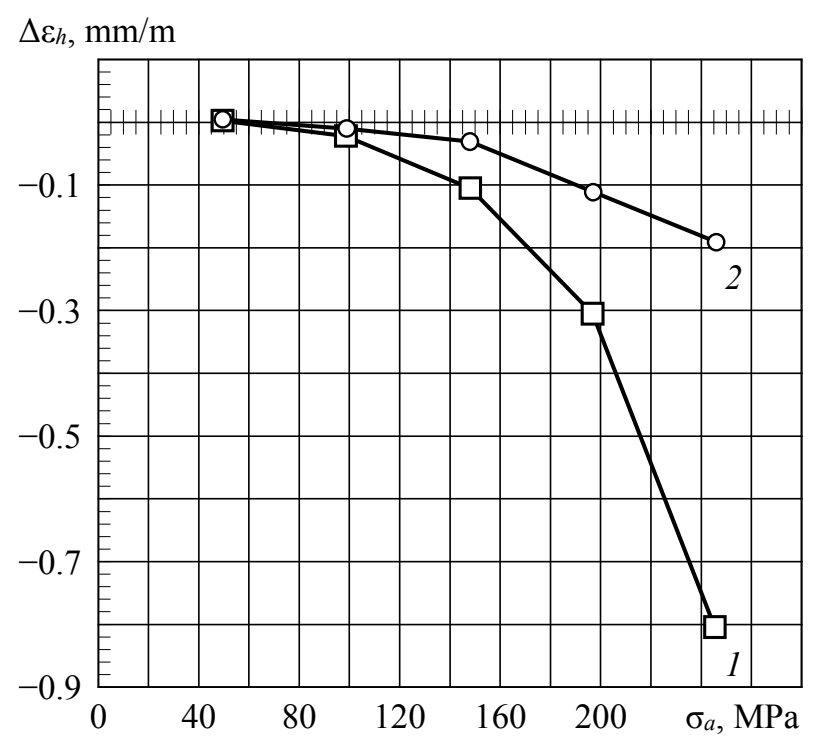

Figure 5. Amplitude dependence of the hysteresis part of disclosure of the inelasticity loop of one of the T700 carbon fiber specimens loaded with a symmetrical cycle with a stepwise increasing amplitude as a transition from tension to compression and back: 1 - inelastic strain in the first cycle, 2 inelastic strain in the second cycle with subsequent stabilization.

The structural elements of the material model naturally describe a large inelastic strain in the first cycle with increasing amplitude. In subsequent loading cycles, the process is stabilized, and we see two areas with enhancement of the hysteresis loop disclosure. Usually, composites have a more pronounced accumulation of residual compression strains if the fibers were sufficiently stretched during the manufacture of the specimens. Otherwise, the opposite process can be observed, although the fracture occurs due to compression [10].

The prediction of longevity is based on the relationship of 
the failure processes distributed over the material volume with the plastic strain values in each of these statistically homogeneous volumes. This is an inherent element of the structural model of the material. Examples of such relationships of the average rate of inelastic deformation with longevity or the disclosure of the inelastic loop with endurance under variable tensile loads are observed in specimens of both metal alloys and composites [2, 10]. The distinctive feature of our approach is that only part of inelastic strain of the material in each range of loading amplitudes is correlated with the rate of failure. Our interpretation implies that each increase in inelasticity is associated with the appearance of new zones (local volumes) in the material structure, where damage appears and begins to develop. Direct observations of damage development show that the areas of damage occurrence are located at different places of the material structure, depending on the stress value, temperature, nature of loading, and duration of the failure process [13].

For metal alloys and composites that work under tension, these relationships are identical, and longevity can be calculated by using the same models after performing their parametric identification. Under compressive loading, however, the failure processes are fundamentally different. In a metal alloy under cyclic compression, the process of damage accumulation has a decaying character [2]. This reduces the residual longevity if followed by tensile loading. For composites, the opposite is true. Under compression, everything is the same as in metals under tension, but a model with different parameters is required. With continuous alternation of compressive and tensile loads, the influence of the latter can be ignored. Based on these assumptions, the longevity of T800 carbon fiber specimens for cyclic loading with various asymmetry indexes was calculated [10].

\section{Failure Under Conditions of Arbitrary Changes in Temperature and Load}

The calculated and experimental values of the longevity of AK4-1 T1 alloy specimens subjected to variable loads and temperatures were compared [2]. The calculations take into account the changes in the material structure as functions of temperature and stresses $[14,15]$. Fracture in this case occurs as a result of cyclic creep of the material occurring on the contour of holes in the specimens. A change in the material structure causes a change in the ratio between the rates of deformation and failure, and the stresses at the strain concentration points are different. Naturally, the longevity of the structure will be affected.

Table 1 shows experimental data and calculations of the longevity of specimens representing a $120 \mathrm{~mm}$ wide strip with a longitudinal stringer riveted along the axis of symmetry. The temperature or its range of variation in the fracture zone $T_{b}$, the nominal stresses $\sigma_{n}$ or their range of changes in the cycle, and the period of the temperature-force loading cycle $t_{c}$ are specified. The range of experimental values of the longevity corresponds to its actual scatter; the range of calculated estimates is obtained taking into account the main errors in reproducing the load and temperature in the experiment. It can be seen that the calculated estimates are in reasonable agreement with the experimental data.

Deviations from the linear dependence of the AEF (Figure 1) require special research, expansion of the temperature-time range of tests, and modeling of structural changes in the material [2]. This will be the next step for solving problems of failure, for example, as a result of thermal fatigue, when temperature and stress are mutually correlated processes.

Duralumin was studied in the widest range of temperature-time loading conditions $[2,11,16]$. A number of experiments conducted at constant stresses and temperatures showed a linear dependence of the AEF on the tensile stresses in the temperature range of $125-200^{\circ} \mathrm{C}$ similar to that shown in Figure 1 for the AK4-1 T1 alloy. Then, by supplementing these data with test results at various strain and loading rates, including cryogenic temperatures, we obtained a fairly comprehensive picture of the behavior of this material. Figure 6 shows the results of thermal activation analysis of all experiments [11].

Table 1. Experimental and calculated estimates of the longevity of a strip with a longitudinal stringer $\left(K_{t}=2.89\right)$ made of AK4-1 T1 alloy under various temperature and force loading programs.

\begin{tabular}{llllll}
\hline \multirow{2}{*}{ Mode } & $\boldsymbol{T}_{b},{ }^{\circ} \mathbf{C}$ & \multirow{2}{*}{$\boldsymbol{\sigma}_{\boldsymbol{n}}, \mathbf{M P a}$} & $\boldsymbol{t}_{\boldsymbol{c}}, \mathbf{s}$ & \multicolumn{2}{l}{ Longevity, $\mathbf{h}$} \\
\hline 1 & & 240 & $17.28-22.28$ & Calculation \\
\hline 2 & & & 120 & $9.9-14.55$ & $5.90-22.7$ \\
3 & $60-135$ & & 480 & $8.2-33.37$ & $5.25-16.28$ \\
4 & & 180 & $12.38-16.12$ & $3.95-24.47$ \\
5 & $83-139$ & $10-310$ & 300 & $8.83-18.47$ & $8.96-32.3$ \\
6 & $38-131$ & & 360 & 10.83 & $12.26-50.86$ \\
7 & $16-127$ & & 360 & $22.63-24.45$ & $10.7-39.6$ \\
8 & $55-135$ & & 90 & 38.87 & $4.38-31.19$ \\
9 & $62-132$ & $290-310$ & 180 & $31.7-40.5$ & $13.68-42.53$ \\
10 & $58-114$ & & 300 & $1.23-1.68$ & $1.49-7.7$ \\
11 & $63-156$ & $10-310$ & - & 0.67 & $0.72-1.64$ \\
12 & 135 & 310 & - & 0.55 & $0.36-0.84$ \\
\hline
\end{tabular}

7 - Mode of curing at $T_{\min }, 8$ - Mode with a constant load at variable temperature stresses. 


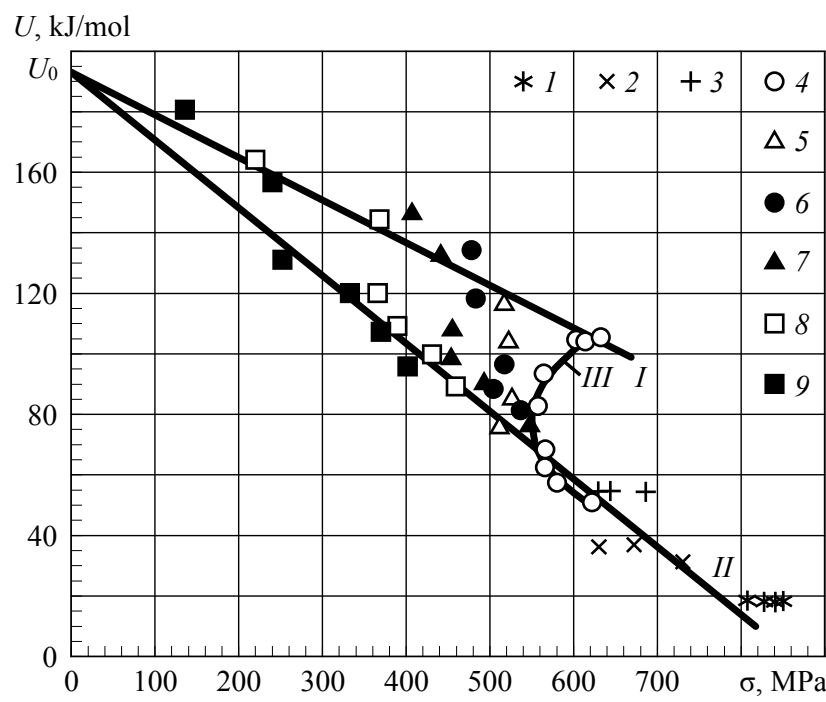

Figure 6. Comparison of force dependences of the AEF of D16 T alloy at constant deformation rates in the range from $10^{-5}$ to $100 \mathrm{~s}^{-1}(293-573 \mathrm{~K})$ with the AEF under monotonous loading (77-293 K); temperature, $K$ : 1 - 77, 2 123, 3-223, 4-293, 5-373, 6-423, 7-473, 8-523, 9-573; I-force dependence of the AEF at constant stresses; II-force dependence of the AEF at srtain rates from 12.5 to $100 \mathrm{~s}^{-1}$ or monotonous loading at cryogenic temperatures for $\approx 30 \mathrm{~s}$; III-dependence of the AEF at $293 \mathrm{~K}$ on the fracture time in the range from 12,500 hours (coinciding with line I) to 0.01-0.001 seconds (coinciding with line II).

The straight line $I$ in the figure represents the force dependence of the AEF corresponding to constant to the constant stresses and temperatures of tests. The remaining values of $U(\sigma)$ are obtained under increasing loads, either at constant strain rates or at constant loading rates. For low temperatures (taking into account the low-temperature features of fracture [11]) and long-term loading at room temperature, the data are given for individual specimens. In other cases, their mean values for several specimens are provided.

As a result, we see the following patterns. If loading occurs slowly (relative to a given temperature), then the AEF values for these loading modes are grouped near line $I$, and the activation volume $\gamma$ has minimum value $\gamma_{\text {min }}$. At high load growth rates, the values of $U(\sigma)$ form another force dependence of the AEF, and $\gamma \gamma_{\text {max }}$. These lines are the boundaries of the transition region from one value of $\gamma$ to the other, which characterizes the change in the material structure associated with the relaxation of internal stresses $[2,3,11,16]$ The same phenomenon is observed in steels, and it becomes clear why their strength characteristics increase at $200-300^{\circ} \mathrm{C}$ [11].

This transition region is modeled by a spectrum of relaxation processes describing the kinetics of changes in internal stresses. Thus, the $\mathrm{PM}_{1}$ and $\mathrm{PM}_{5}$ bodies can have their own internal structure. In the first approximation, we can use its formal description based on the elements of viscous flow, i.e., Maxwell bodies [17]. In this case, several parallel connected Maxwell bodies can replace one $\mathrm{PM}_{5}$ element [2] However, this situation requires a more profound study of internal thermodynamic processes in materials.

The changes in temperature and stresses can be in different proportions. When the rates of their changes are comparable, the solutions of the differential equations for the structural models of the material (3)-(9) are different. For the case of thermo-cyclic loading (Table 1), it is more convenient to use an approximate solution at a time step. Let the plastic flow rates of the material at the beginning and at the end of the time step $\Delta t$ with allowance for the structural changes in the material be

$$
\dot{\varepsilon}_{1}=\varepsilon_{*} v_{0} \exp \left(-\frac{Q_{0}-\alpha_{1} \sigma_{1}}{R T_{1}}\right)
$$

and

$$
\dot{\varepsilon}_{2}=\varepsilon_{*} v_{0} \exp \left(-\frac{Q_{0}-\alpha_{2} \sigma_{2}}{R T_{2}}\right)
$$

The change in the plastic strain rate at the step is represented as

$$
\dot{\varepsilon}=\widehat{A} \exp (\widehat{B}+\hat{D} \Delta t)
$$

assuming the argument under the exponent to change linearly. Then, after integration we obtain, e.g., the plastic strain component in solution (5):

$$
\Delta \varepsilon=\widehat{A} \exp (\widehat{B}) \frac{\exp (\widehat{D} \Delta t)-1}{\widehat{D}} .
$$

In fatigue failure, the stresses usually change much faster than the temperature, and it can be considered as constant at a time step and as stepwise changing from step to step. As the material model describes the temperature dependence of inelasticity, it is possible to recalculate the fatigue failure data to a different temperature even without having any experimental data. For example, at the design stage, one has to evaluate the load conditions allowed for the resource, when the material properties for this case have not yet been studied. An example of such a calculation is given below.

Shabalin conducted fatigue tests of the same specimens, whose strength characteristics at constant stresses are shown in Figure 1 [18]. The specimens were loaded with a frequency of $6.67 \mathrm{~Hz}$ at temperatures of 20 and $150^{\circ} \mathrm{C}$. For each test temperature, two loading modes were selected, differing in endurance by about three times and with approximately the same values in both cases.

As the experimental data for parametric identification of the material model were available in the required volume only for the $1201 \mathrm{~T} 1$ alloy, the calculations were performed on the basis of the model of this material. Therefore, the problem is formulated as follows: how much should the loading amplitude reduced for the longevity (or endurance) to remain unchanged with an increase in temperature? The calculations were performed using 64 time steps in a period of the 
harmonic loading cycle, assuming a linear transition of external stresses from one value to another within the time step. The results of the experiment and calculations are shown in Table 2 [2]. The experimental data are represented by mean logarithmic values of endurance; the results of longevity calculations are obtained for the fracture probability of 0.5 and then recalculated into the number of cycles.

Table 2. Comparison of experimental and calculated values of endurance of specimens tested at temperatures of 20 and $150^{\circ} \mathrm{C}$.

\begin{tabular}{lllll}
\hline \multirow{2}{*}{$\boldsymbol{T}, \mathbf{K}$} & \multicolumn{2}{l}{ Experiment, AK4-1T 1alloy } & \multicolumn{2}{l}{ Calculation, 1201T1 alloy } \\
\cline { 2 - 5 } & $\boldsymbol{\sigma}_{\boldsymbol{a}}, \mathbf{M P a}$ & $\boldsymbol{N}$, cycle & $\boldsymbol{\sigma}_{\boldsymbol{a}}, \mathbf{M P a}$ & $\boldsymbol{N}$, cycle \\
\hline \multirow{2}{*}{293} & 130 & 165,700 & 100 & 164,287 \\
& 150 & 46,040 & 120 & 47,514 \\
\multirow{2}{*}{423} & 120 & 151,000 & 90 & 154,055 \\
& 140 & 48,920 & 110 & 46,391 \\
\hline
\end{tabular}

A comparison of the calculation and experiment shows that the loading amplitude has to be reduced by $10 \mathrm{MPa}$ for the longevity (or endurance, as they are proportional in this case) to remain approximately the same. If the loading frequencies differ significantly, then the material model will provide the value of longevity because the structures are loaded and destroyed over time. By the example of experiment and calculations, we demonstrate how the mathematical model describes what we observe in reality.

The specimens whose fatigue characteristics are shown in Figure 3 were tested at three frequencies of $0.1,1$, and $40 \mathrm{~Hz}$ with a symmetric loading cycle and with the same amplitude of $80 \mathrm{MPa}$. Moreover, at a frequency of $1 \mathrm{~Hz}$, additional tests were carried out with a triangular shape of the loading cycle. The experimental and calculated results are summarized in Table 3. The actual range of endurance and its mean logarithmic value are specified. The calculations were also performed for the fracture probability of 0.5 at time steps and then recalculated into the number of cycles.

Table 3. Comparison of experimental and calculated values of endurance of specimens from the 1201 AT1 alloy sheet with a cross section of $6 \times 30$ mm with a central hole of $\varnothing 20 \mathrm{~mm}\left(K_{t}=2.08\right)$, tested at different frequency and shape of the cycle $\left(20^{\circ} \mathrm{C}\right)$.

\begin{tabular}{llll}
\hline \multirow{2}{*}{ Cycleshape, frequency } & Experiment: $\boldsymbol{\sigma}_{\boldsymbol{m}}=\mathbf{0}, \boldsymbol{\sigma}_{\boldsymbol{a}}=\mathbf{8 0} \mathbf{M P a}$ & $\left\langle\boldsymbol{N}_{\mathbf{l o g}}\right\rangle$, cycle \\
\cline { 2 - 3 } & $\boldsymbol{N}$, cycle & 128,189 \\
Sinus, $40 \mathrm{~Hz}$ & $99,900-272,900,9$ specimens & 122,661 & 127,678 \\
Triangle, $1 \mathrm{~Hz}$ & $116,672-128,176,3$ specimens & 118,190 & 127,160 \\
Sinus, $1 \mathrm{~Hz}$ & $106,420-140,890,5$ specimens & 104,272 \\
Sinus, $0.1 \mathrm{~Hz}$ & $73,710-122,990,5$ specimens & 121,453 \\
\hline
\end{tabular}

Comparing the experimental and calculated data, we see that the mathematical model tracks the time factors of loading. If these data are represented graphically, the patterns become obvious: endurance changes approximately in proportion to the change in the logarithm of frequency, and longevity is almost inversely proportional to frequency. This follows from the solutions of differential equations for the flow of $\mathrm{PM}_{1}$ or $\mathrm{PM}_{5}$ bodies under rigid (in terms of strain) loading [5]. This is evidenced by solutions (3), (7), and (8): changes in strain or stress occur with a change in the logarithm of the loading or strain rate. Therefore, an increase in frequency induces only a minor change in the damage in the cycle and an almost proportional decrease in the longevity. The triangular shape of the cycle leads to greater longevity because there is no time delay at maximum stress. This is what we should be interested in.

Mathematical modeling of deformation and failure processes allows one to avoid schematization of initial information about the loads based on the "ideology of the cycle," when it comes to fatigue. In addition, the operation of structures occurs at a variable temperature, which significantly affects the longevity of composites. For example, we have a spectrum of loads in operation, represented by the spectral density distribution over the frequency. The longevity can be estimated directly from the process realization. If there are statistical data on such processes, then the equivalent pseudorandom process (PRP) with the same statistical characteristics is synthesized from the averaged spectral density. Then, according to its realization, a calculated estimate is obtained.

Tests were conducted at different loading spectra with simulation of a real random process by various types of PRPs on BiSS $100 \mathrm{kN}$ (Bangalore Integrated System Solutions) and MTS-10 testing machines. The registered loads in the form of the bending moment of the wing of a transport aircraft were taken as the basis [19]. The estimates for the specimens with a cross section $6 \times 30 \mathrm{~mm}$ with a central hole $\varnothing 5 \mathrm{~mm}$ made of $1201 \mathrm{~T} 1$ alloy tested under different loading spectra with a standard deviation (SD) of $47 \mathrm{MPa}$ and two mean stress values $\left(\sigma_{m}=0\right.$ and $\left.100 \mathrm{MPa}\right)$ are compared in Figure 7 . The calculation was based on the effective strain concentration factor for fatigue $K_{f}=1+q\left(K_{t}-1\right)$ [20] corresponding to the quality of the material (parameter $q$ ) at the time of delivery of semi-finished products (forged-rolled plates) [2], where $K_{t}$ is the theoretical stain concentration factor.

Experiments 2, 3, 5 and 6 simulate the same spectrum of real loading in the frequency range from 0.08 to $16 \mathrm{~Hz}$. Experiments 7 and 8 represent the spectrum of narrow-band random noise in the loading frequency range from 0 to $5.5 \mathrm{~Hz}$ [2]. In addition, experiment 9 was conducted, in which the same spectrum was represented by harmonics distributed over the frequency logarithm. Thus, the dispersion was displayed in more detail at low frequencies of the spectrum and more roughly at high frequencies. The SD and $\sigma_{m}$ were chosen the same in order to see the dependence of the longevity on the discreteness and frequency composition of spectra.

The difference between the spectra from each other is that the frequency range was limited to the frequency of $5.5 \mathrm{~Hz}$ in the case with 13 harmonics in the spectrum of narrow-band random noise. With 62 harmonics distributed over the frequency logarithm, the high-frequency part of the spectrum is also represented by a small dispersion value. Therefore, despite the small dispersion in the high-frequency part of the 
loading spectrum, which is not presented in much detail, its effect on the fracture time is very significant. Even with a slight simplification in the representation of the high-frequency part of the spectrum (experiment 9), there is already an increase in longevity.

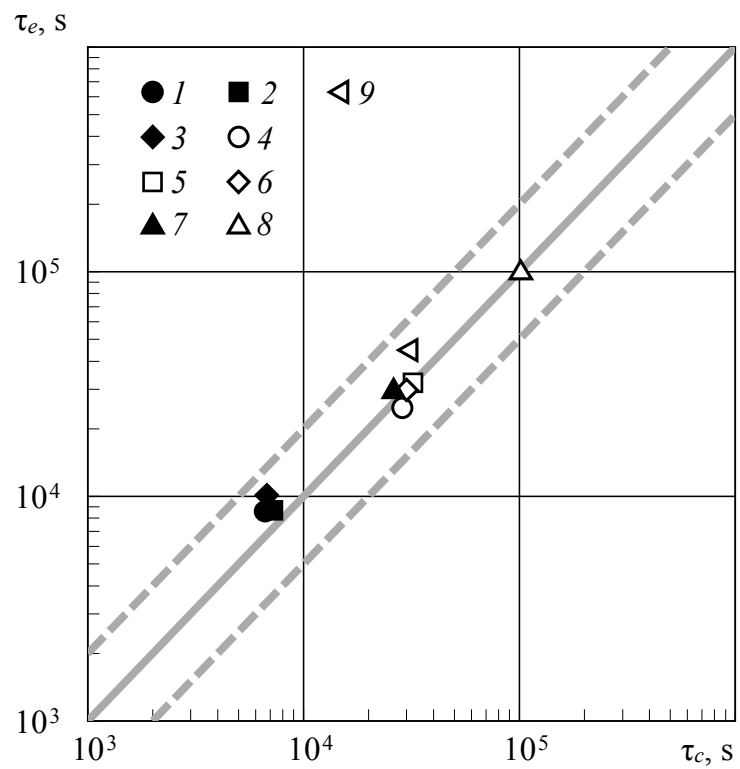

Figure 7. Comparison of calculated longevity estimates $\tau_{\mathfrak{c}}$ for different loading spectra with experimental logarithmic mean values of the longevity $\tau_{\mathrm{e}}$ of the 1201 T1 alloy specimens: 1 - real spectrum of loading and $2-P R P$ of 67 harmonics $\left(\sigma_{m}=100 \mathrm{MPa}\right), 3-\mathrm{PRP}$ of 23 harmonics $\left(\sigma_{m}=100 \mathrm{MPa}\right), 4-$ real spectrum of loading and $5-P R P$ of 67 harmonics $\left(\sigma_{m}=0\right), 6-P R P$ of 23 harmonics $\left(\sigma_{m}=0\right), 7-P R P$ of 13 harmonics $\left(\sigma_{m}=100 \mathrm{MPa}\right), 8-P R P$ of 13 harmonics $\left(\sigma_{m}=0\right), 9-P R P$ of 62 harmonics, distributed according to the logarithm of frequency $\left(\sigma_{m}=0\right)$.

Program tests of T800 carbon fiber specimens were also performed. The calculated values of their endurance under harmonic loading with a frequency of 3 or $5 \mathrm{~Hz}$ and a negative asymmetry index were previously compared with the experiment [10]. The forced loading program was a conditional cycle scheme for the upper panel of the aircraft wing, displaying a supposedly "equivalent" flight cycle, and compressive stresses were dominating on the average. In the experiment and in the calculation, this program was reproduced in time with block duration of $8.5 \mathrm{~s}$ at a frequency of $1 \mathrm{~Hz}$ (Figure 8).

The mathematical model of the material calculated the process of fatigue failure of the composite also by time steps in accordance with its time realization in the experiment. The calculated longevity estimates converted into cycles and blocks of program loading are compared in Figure 9, including the previous data.

The model calculations agree reasonably with experimental data for various loading asymmetries, including different frequency and shapes of the loading cycles. The issue of equivalence of design testing programs requires separate consideration. From the viewpoint of mathematical modeling, if the longevity of a certain place in the structure under loading in operation and in tests is determined by the same structural element of the material model, then the equivalent is correctly characterized by the ratio of the failure times. Otherwise, with a forced loading program, it is necessary to calculate it by the damage accumulated by the element of the model that determines the longevity in operation.

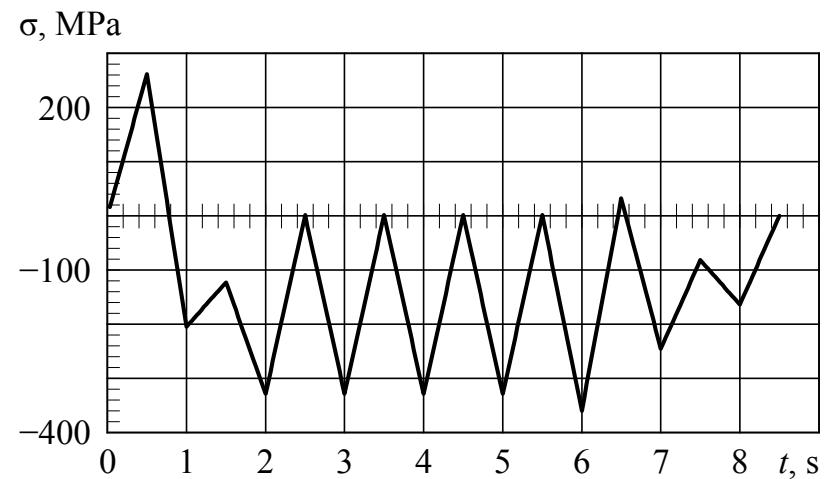

Figure 8. Program of loading for conditional flight realization of the process.

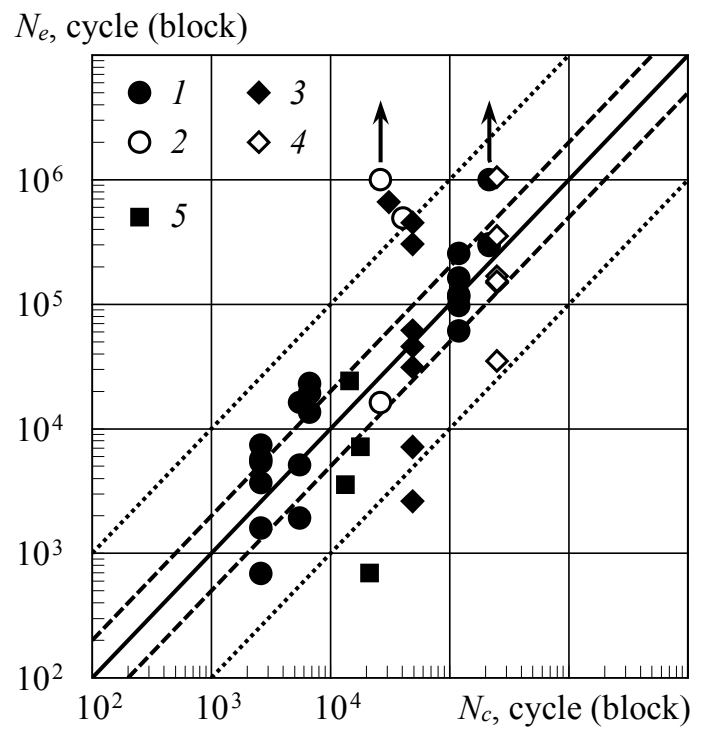

Figure 9. Comparison of calculated $N_{c}$ and experimental $N_{e}$ endurance values carbon fiber specimens tested with different loading asymmetries (actual scatter in the experiment); $\sigma_{m}, M P a: 1-0,2--50,3--110,4--166.5,5-$ -47.75 (program tests).

\section{Criterion of Fracture}

The rate of fracture is understood to be the inverse of longevity,

$$
\dot{\omega}=\frac{1}{\tau}=v_{0} \exp \left(-\frac{U_{0}-\gamma \sigma}{R T}\right)
$$

for bodies with the $\mathrm{Zh}$ elements or

$$
\dot{\omega}=v_{0} \exp \left(-\frac{U_{0}}{R T}\right)\left[\exp \left(\frac{\gamma \sigma}{R T}\right)-\exp \left(-\frac{\gamma \sigma}{R T}\right)\right]
$$

for bodies with the $\mathrm{Km}$ elements, if the loading conditions and material structure are constant. In general, the fracture condition 
(appearance of crack or delamination) is expressed as

$$
\int_{0}^{\tau} \dot{\omega}(t, \sigma, T) \mathrm{d} t=1,
$$

which satisfies the Bailey criterion [21]. This condition implies that the threshold concentration of damage is reached in some volume of the solid body. Expression (10) corresponding to numerous experimental data is also confirmed by a numerical experiment performed by the molecular dynamics method [22].

The rate of fracture is associated with the rate of the plastic flow of the $\mathrm{Zh} \mathrm{(1)} \mathrm{or} \mathrm{Km} \mathrm{(2)} \mathrm{bodies.} \mathrm{The}$ dependence of the rate of fracture in (12) material structure, reflected in the structural elements of the $\mathrm{PM}_{1,5}$ and $\mathrm{PM}_{2,6}$ model. Independent summation of damages over the structural elements of the material model ensures better agreement of the calculation with the experiment than their summation in one measure [2]. The same thing is observed in structure testing. The place of the most rapid failure of the structure depends on the value of the loading amplitude.

According to Kauzmann, expression (2), as well as (11), takes into account the reverse flow through the potential barrier with the same probability in both forward and reverse directions. He was the first to apply the theory of reaction rates to the flow of solids [23]. In reality, these probabilities may differ, and some very small value of safe stresses is found in experiments [6]. Damages do appear and accumulate, but their concentration is insufficient for macro-fracture of the solid.

The operation of the concentration criterion of fracture is illustrated by the pictures taken with an atomic-force microscope during glass failure [24]. When the sizes of pores become comparable with the distance between them, their coalescence occurs, and this leads to crack propagation.

\section{Conclusion}

Thus, mathematical modeling of the processes of failure and plastic flow of the material in individual zones distributed over its volume allows obtaining correct estimates of the longevity of both metal alloys and composites, including cases of arbitrary temperature and force loading. Thus, the use of artificial techniques that reduce reliability of these estimates can be avoided.

There are fundamental differences in the behavior of metal alloys and composite materials under tension and compression, and different mathematical models are required for calculating the estimates of longevity with the prevalence of loads of this or that type.

\section{Acknowledgements}

The author is grateful to S. A. Katarushkin, as well as to many other colleagues who helped him in numerous laborious experiments.

\section{References}

[1] D. A. Gokhfeld and O. F. Chernyavskii. Bearing capacity of structures under repeated loads. Moskva (USSR): Mashinostroenie, 1979. Russian.

[2] M. G. Petrov. Strength and life of structural components: an approach based on model of material as a physical medium. Saarbrücken (DE): Lambert Academic Publishing; 2015. Russian.

[3] V. A. Stepanov, N. N. Peschanskaya, V. V. Shpeizman, and G. A. Nikonov (1975). Longevity of solids at complex loading. International Journal of Fracture, 11 (5): 851-867.

[4] A. R. Michetti (1977). Fatigue analysis of structural components through math-model simulation. Experimental mechanics, 2: 69-76.

[5] M. G. Petrov (1998). Rheological properties of materials from the point of view of physical kinetics. Journal of Applied Mechanics and Technical Physics, 39 (1): 104-112.

[6] V. A. Petrov, A. Ya. Bashkarev, V. I. Vettegren. Physical foundations for predicting the longevity of structural materials. S.-Peterburg (Russia): Politekhnika, 1993. Russian.

[7] L. N. Stepanova, M. G. Petrov, V. V. Chernova, V. L. Kozhemyakin, and S. A. Katarushkin (2016). Investigation of the inelastic properties of carbon fiber during cyclic testing of specimens using acoustic emission and tensometry methods. Deformation and fracture of materials, 5 : 37-41. Russian.

[8] L. N. Stepanova, M. G. Petrov, V. V. Chernova (2017). Acoustic emission control of inelastic properties of carbon fiber reinforced plastic with various reinforcement schemes under cyclic loading. Control. Diagnostics, 8: 18-25. Russian.

[9] Yu. N. Rabotnov. Creep of structural components. Moskva (USSR): Mashinostroenie, 1966. Russian.

[10] M. G. Petrov. Numerical simulation of fatigue failure of composite materials under compression. V. Fomin and L. Placidi (Eds.). EPJ Web of Conferences Volume 221 (2019): XXVI Conference on Numerical Methods for Solving Problems in the Theory of Elasticity and Plasticity (EPPS-2019 Tomsk, Russia, June 2019).

[11] M. G. Petrov (2016). Fundamental studies of strength physics methodology of longevity prediction of materials under arbitrary thermally and forced effects. International Journal of Environmental and Science Education, 11 (17): 10211-10227.

[12] A. S. Krausz and H. Eyring. Deformation kinetics. New York (NY): John Wiley and Sons, 1975.

[13] Yu. F. Balandin. Thermal fatigue of metals in marine power engineering. Leningrad (USSR): Sudostroenie, 1967. Russian.

[14] M. G. Petrov (1976). A relationship between the strain and failure of stressed solids. Journal of Applied Mechanics and Technical Physics, 7 (6): 853-856.

[15] M. G. Petrov. Decay of metallic supersaturated solid solutions at the fracture of precipitation hardening aluminum alloys. Proceedings of the VI International scientific school-conference "Fundamental and applied materials science.” Barnaul (Russia): Altay STU. 2009, pp. 203-209. Russian. 
[16] M. G. Petrov and A. I. Ravikovich (2001). Kinetic approach to prediction of the life of aluminum alloys under various thermal-temporal loading conditions. Journal of Applied Mechanics and Technical Physics, 42 (4): 725-730.

[17] A. S. Nowick and B. S. Berry. Anelastic relaxation in crystalline solids. New York (NY), London (GB): Academic Press, 1972.

[18] V. I. Shabalin. Investigation of fatigue of metals at stresses above the yield point [dissertation]. Moskva (USSR): VIAM, 1970. Russian.

[19] M. G. Petrov. On estimation of damages of aircraft structures in operation. High Energy Processes in Condensed Matter 2019. AIP Conference Proceedings 2125. (accessed 2019 Sep 11). https://aip-info.org/2FAM-1G5WC-8XI93L-XGDN5-0/c.aspx
[20] J. A. Collins. Failure of materials in mechanical design: analysis, prediction, prevention. New York (NY): John Wiley and Sons, 1981.

[21] J. Bailey (1939). An attempt to correlate some tensile strength measurements on glass. Glass Industry, 20: 21-25.

[22] V. S. Yuschenko and E. D. Schukin (1981). Molecular dynamics modeling in studying mechanical properties. Physical and Chemical Mechanics of Materials, 4: 46-59. Russian.

[23] W. Kauzmann (1941). Flow of solid metals from the standpoint of the chemical-rate theory. Transactions of the AIME, 143: $57-83$.

[24] F. Célarié, S. Prades, D. Bonamy, L. Ferrero, E. Bouchaud, C. Guillot, and C. Marlière (2003). Glass breaks like metal, but at the nanometer scale. Physical Review Letters, 90 (7): 075504. 